\title{
La dosimétrie cytogénétique de l'accident d'irradiation
}

\author{
P. VOISIN ${ }^{\prime}$, M. BENDERITTER ', V. CHAMBRETTE ${ }^{1}$, M. CLARAZ ${ }^{1}$, \\ M. DELBOS ${ }^{\prime}$, V. DURAND ${ }^{1}$, N. PAILLOLE ${ }^{1}$, L. ROY $^{1}$, \\ I. SOROKINE-DURM ${ }^{1}$
}

(Manuscrit reçule 30 mars 2001, accepté le 29 juin 2001)

RÉSUMÉ Lors d'une suspicion d'exposition accidentelle aux rayonnements ionisants, la détermination de la dose reçue au moyen de paramètres biologiques est une part importante de la stratégie thérapeutique, en complément des signes cliniques et de la dosimétrie physique. Le dénombrement des aberrations chromosomiques instables dans les lymphocytes du sang périphérique est aujourd'hui la méthode de référence. La préparation des échantillons biologiques dépend cependant du but à atteindre, de l'expertise précise de suspicions d'irradiations ou du tri rapide en cas d'accident de grande envergure. Une adaptation peut être nécessaire s'il s'agit d'irradiation hétérogène ou ancienne. Malgré cette robustesse et ces adaptations, la cytogénétique conventionnelle reste une technique lourde, longue, réservée à du personnel spécialisé. Le dénombrement des micronoyaux dans les lymphocytes binucléés peut constituer une alternative, apparemment plus facile et plus simple que le test des dicentriques. À partir de l'expérience acquise par I'IPSN ces dernières années sur l'expertise des suspicions d'irradiation, ce papier a pour but de dresser un bilan technique quoique succinct de ces différentes approches telles que nous les avons adaptées aux suspicions d'irradiation récente.

ABSTRACT The cytogenetics biodosimetry of accidental overexposure.

For a suspicion of accidental exposure to the ionizing radiations, the determination of the dose received by means of biological parameters is a significant part of the therapeutic strategy, in complement of the clinical signs and physical dosimetry. The scoring of the unstable chromosome aberrations in peripheral blood lymphocytes remains the reference method. The preparation of the biological samples depends however on the goal to reach, the precise expertise of few irradiations or the rapid sorting in the event of a large-scale accident. An adaptation can be necessary in case of heterogeneous or past exposure. In spite of this robustness and these adaptations, the conventional cytogenetics remains a tedious, time-consuming technique, reserved to specialized personnel. The scoring of the micronuclei in the binucleated lymphocytes can constitute a credible alternative to the dicentrics assay, apparently easier and quicker. From the experience gained by the IPSN these last years on the expertise of irradiation suspicions, the purpose of this paper is to draw up a brief technical assessment of these various approaches such as adapted to suspicions of recent overexposure.

\footnotetext{
'Institut de protection ct de sûreté nucléaire, BP 6, 92265 Fontenay-aux-Roses Cedex, France.
} 


\section{Introduction}

Lorsqu'un individu a récemment subi une surexposition accidentelle aux rayonnements ionisants, la connaissance de la dose reçue au niveau de l'organisme est une des informations utiles aux équipes hospitalières pour asseoir la stratégie thérapeutique la plus appropriée.

Une irradiation de 1 Gy et plus se traduit par des signes cliniques précoces (nausée, diarrhée) qui permettent une première évaluation de la dose reçue par l'accidenté. Cette évaluation peut être complétée par une dosimétrie physique qui se fonde sur l'analyse de dosimètres externes portés par le personnel à risque ainsi que sur la reconstitution physique de l'accident (activité de la source, durée de l'exposition) spécialement nécessaire pour les personnes du public ayant été accidentellement exposées. Dans le but de connaître l'atteinte au niveau de l'individu lui-même et de reconstituer une dose à la suite d'un accident d'irradiation, il est en plus utile de compléter l'estimation de la dose physique et clinique par l'étude des variations radio-induites de certains paramètres biologiques. Seule cette dosimétrie biologique peut rendre compte de la radiosensibilité propre à l'individu concerné.

Le dénombrement des aberrations chromosomiques de type instable (dicentriques, anneaux centriques) dans les lymphocytes du sang périphérique est considéré encore aujourd'hui comme la méthode la plus spécifique et la plus sensible pour une irradiation récente (Bender et al., 1988 ; Du Frain et al., 1980 ; IAEA, 1986). Elle a d'ailleurs acquis en France comme dans d'autres pays une valeur médico-légale. Ainsi, durant la période 1992-1999, le laboratoire de dosimétrie biologique de l'IPSN a été amené à se prononcer sur environ 200 suspicions d'irradiation, nombre de cas suffisant pour amener certaines constatations et tirer quelques conclusions.

Dans le cas d'une suspicion d'irradiation impliquant peu de personnes $(<10)$, et compte tenu des circonstances de l'accident radiologique, la qualité des préparations et la précision de l'estimation doivent être privilégiées. En cas d'accident impliquant un grand nombre d'irradiés, les incertitudes de la dosimétrie physique et même clinique augmentent en proportion du nombre d'individus potentiellement irradiés, ce qui justifie encore l'intérêt d'une dosimétrie biologique à titre individuel. Son utilité n'apparaît cependant pleinement que si l'estimation de la dose peut être rendue dans les heures, sinon les jours qui suivent l'irradiation réelle ou supposée. La précision de la mesure et la qualité des préparations apparaissent en proportion moins importante que le délai de réponse, ce qui nécessite une procédure particulière. 
Néanmoins, la cytogénétique conventionnelle reste une technique lourde et surtout limitée à des personnels qualifiés en cytogénétique. Il existe une autre technique cytogénétique plus simple et potentiellement plus rapide qui, dans le cas d'un tri, pourrait remplacer utilement la cytogénétique conventionnelle. Le dénombrement des micronoyaux dans les lymphocytes du sang périphérique est une méthode de plus en plus utilisée pour mettre en évidence les dommages induits par certains facteurs génotoxiques (Muller et Streffer, 1994 ; Paillole et Voisin, 1998). Quoique la phase de préparation des échantillons sanguins soit légèrement plus longue que pour les dicentriques, le dénombrement des micronoyaux dans les lymphocytes binucléés est plus simple et plus rapide. Le principal problème du test des micronoyaux est son manque de spécificité pour les facteurs clastogènes. II faut donc vérifier si son utilisation est acceptable en situation accidentelle et particulièrement pour un accident de large envergure.

Ce papier a pour but de dresser un bilan technique quoique succinct de ces différentes approches telles que nous les avons adaptées aux suspicions d'irradiation récente.

\section{Matériel et méthodes}

\subsection{Dénombrement des aberrations chromosomiques instables}

Le sang est prélevé stérilement par ponction veineuse antécubitale sur héparinate de lithium dans les heures, les jours qui suivent l'accident. Les cultures de sang total sont effectuées suivant une procédure similaire à celle préconisée par le rapport technique de l'AIEA (1986) et publiée par ailleurs (Voisin et al., 1996). En bref, $0,5 \mathrm{ml}$ de sang est ajouté dans un tube à un milieu de culture approprié (RPMI 1640, Life Technologies), additionné d'un agent mitogène, la phytohémaglutinine (Life Technologies), de plusieurs antibiotiques et de $10 \%$ de sérum de veau fotal (SVF, Life Technologies). Les cultures sont effectuées le jour même, en double. Par ailleurs, des cultures supplémentaires sont effectuées le lendemain, pour des problèmes éventuels de contamination, de mauvaises manipulations ou de mauvaises conditions de culture. Un analogue de la thymidine, la 5-bromodeoxyuridine (BrdU, Sigma), est également ajouté à chaque tube de culture.

Après 46 heures de culture à $37^{\circ} \mathrm{C}$, un inhibiteur mitotique, la démécolcine (Sigma), est ajouté dans les tubes, et les cultures prolongées de 2 à 3 heures. Les lymphocytes sont séparés par centrifugation, et un choc hypotonique au $\mathrm{KCl}$ $(0,075 \mathrm{M})$ est pratiqué pour lyser les hématies et gonfler les lymphocytes. Après centrifugation, les cellules sont fixées par addition de méthanol/acide acétique $(3 / 1, v / v)$. Les métaphases sont étalées par projection sur des lames de microscope 
propres et humides, et les premières mitoses sont visualisées par la technique FPG (Perry et Wolf, 1974). L'observation rapide des lames des premières cultures permet de repérer les cellules présentant un délai mitotique plus important: les cultures supplémentaires sont alors prolongées de quelques heures.

Après coloration, les métaphases sont examinées au microscope et les anomalies chromosomiques instables (dicentriques, anneaux centriques et fragments) dénombrées seulement dans les premières mitoses «complètes » à 46 centromères.

\subsection{Dénombrement des micronoyaux}

Le test des micronoyaux peut être pratiqué à partir d'une aliquote de $150 \mu \mathrm{l}$ de sang pour chaque tube de culture cellulaire (Muller et Streffer, 1994). Les autres conditions de culture restent globalement identiques à la cytogénétique conventionnelle, si ce n'est la durée de la culture qui est allongée à 64 heures et si ce n'est l'addition de $25 \%$ de sérum de veau fœtal. De la cytochalasine B (Sigma) est ajoutée au milieu de culture après 40 heures, pour bloquer un maximum de lymphocytes au début de la seconde interphase. Les lymphocytes sont séparés par centrifugation, et un choc hypotonique au $\mathrm{KCl}(0,125 \mathrm{M})$ est pratiqué pour lyser les hématies et gonfler les lymphocytes. Après centrifugation, les cellules sont fixées par addition de méthanol/acide acétique $(6 / 1, v / v)$. Les cellules sont étalées par projection sur des lames de microscope propres et observées en fluorescence, après addition d'iodure de propidium, un colorant spécifique de l'ADN. Les micronoyaux sont dénombrés dans les lymphocytes binucléés uniquement.

\section{Résultats}

\subsection{Courbes dose-effet et précision de la dose estimée}

L'estimation de la dose nécessite l'établissement de courbes dose-effet de référence. La variation de la fréquence des aberrations chromosomiques instables ou celle des micronoyaux en fonction de la dose peut être modélisée par l'équation générale suivante $Y=\alpha D+\beta D^{2}+$ cte, où $D$ représente la dose d'irradiation reçue et $Y$ la fréquence des aberrations (IAEA, 1986). Les facteurs $\alpha$ et $\beta$ représentent les coefficients d'ajustement de la courbe et «cte » représente la fréquence spontanée d'aberrations dans une population normale. Ces coefficients sont calculés à partir des points expérimentaux de la courbe en effectuant un ajustement itératif du maximum de probabilité. Suivant le type de particules ionisantes à l'origine des aberrations chromosomiques, les valeurs des coefficients et donc l'allure de la courbe varient. 


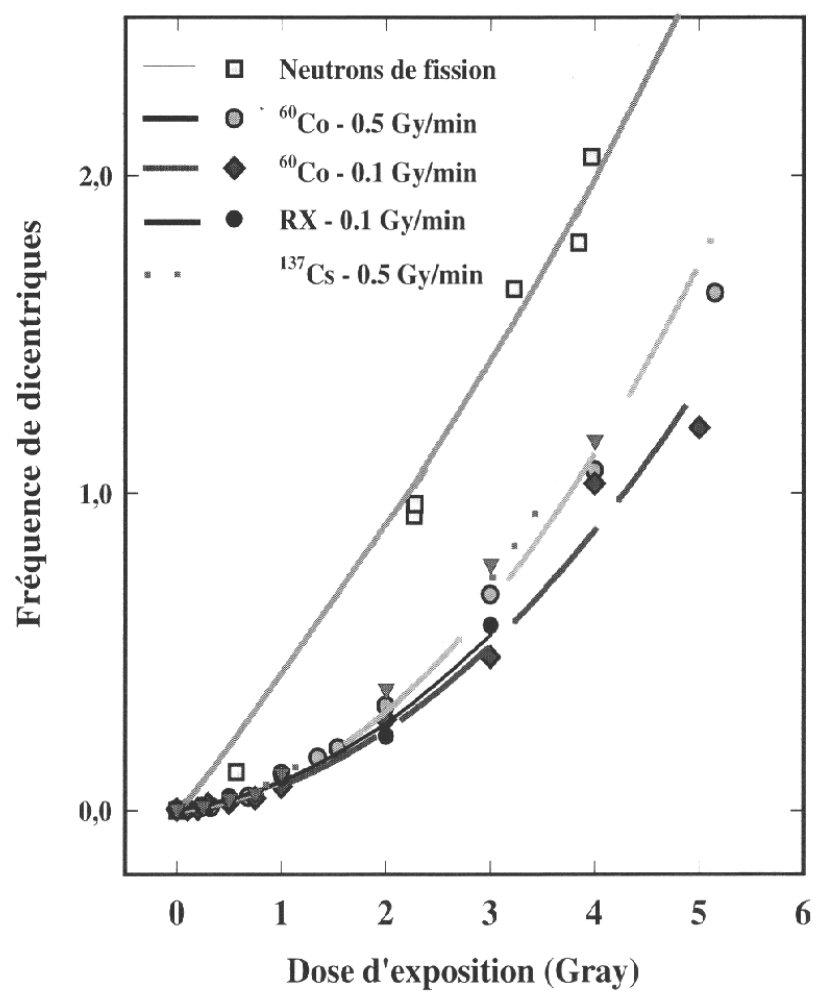

Figure 1 - Relations dose-effet établies en dénombrant les aberrations chromosomiques induites dans les lymphocytes du sang périphérique par des rayonnements ionisants de différentes qualités et à différents débits de dose.

Dose-effect relationships fitted by scoring unstable chromosome aberrations induced in blood peripheral lymphocytes by ionizing radiation of different qualities and of various dose rates.

La pente initiale de la courbe est d'autant plus faible que le débit de dose est réduit. Par contre, pour les neutrons ou plus généralement les rayonnements de TEL élevé, la relation tend à être linéaire. De plus, la pente est plus élevée que celle des rayonnements $\mathrm{X}$ et $\gamma$, traduisant une production d'aberrations plus importante. Le nombre d'aberrations paraît alors directement proportionnel à la dose, sans effet notable du débit de dose. Ainsi au cours d'un accident de criticité, où le flux est généralement mixte (neutrons $+\gamma$ ), les effets biologiques dus aux neutrons sont prédominants à dose équivalente sur ceux dus aux rayons $\gamma$. La connaissance de ces effets relatifs peut se révéler essentiel dans le traitement d'éventuels accidents d'irradiation. 
Les courbes rassemblées sur la figure 1 sont établies à partir de la fréquence des dicentriques seuls. Il est d'usage dans certains laboratoires d'établir ces courbes en utilisant la fréquence des dicentriques et des anneaux centriques. La forme globale reste néanmoins la même, car l'expérience montre que la production d'anneaux centriques représente $1 / 10$ de la fréquence des dicentriques radio-induits.

Contrairement à la formation des dicentriques, la formation des micronoyaux est une conséquence indirecte des effets des rayonnements ionisants, puisque les micronoyaux résultent d'une élimination de fragments ou de chromosomes entiers par la cellule. Le manque de spécificité des micronoyaux induit une grande variabilité des résultats d'un individu à l'autre, toute autre caractéristique (âge, sexe, ...) étant constante par ailleurs. Dans un premier temps, nous avons cherché à déterminer si cette variabilité individuelle, notamment aux faibles doses, était réellement un handicap en cas d'accident radiologique. Une courbe dose-effet a été établie à partir de sang prélevé sur 47 donneurs sains d'âge compris entre 25 et 30 ans, irradié par le rayonnement $\gamma \mathrm{du}{ }^{60} \mathrm{Co}$ entre 0 et 4 Gy à un débit de dose de $0,5 \mathrm{~Gy} \mathrm{~min}^{-1}$, ce qui constitue à notre connaissance, la première relation dose-effet établie sur autant d'individus. Ce travail a montré que la reproductibilité des mesures était suffisante pour obtenir une courbe de référence utilisable pour l'estimation des doses. Cependant, la fréquence des micronoyaux spontanément induits ne permet pas d'estimer de doses au-dessous de 0,32 Gy. En ce qui concerne les courbes de référence, on constate que les effets globaux (forme des courbes, influence du TEL, du débit de dose) décrits pour les dicentriques s'appliquent aussi aux micronoyaux (Paillole et Voisin, 1998).

\subsection{Application de la cytogénétique conventionnelle aux suspicions d'irradiation accidentelle}

Le sang est prélevé par le médecin en charge du patient potentiellement irradié dans les heures, voire les jours qui suivent l'exposition. L'expertise de la dose est effectuée à partir du dénombrement des dicentriques dans 500 métaphases complètes. La précision statistique attendue de la mesure $( \pm 0,2$ Gy d'intervalle de confiance à $95 \%$ ) paraît satisfaisante par rapport au temps passé ( 2 jours/technicien).

Les différents cas de suspicion d'irradiation concernent deux grandes catégories de personnes (Voisin et al., 1996) :

(a) les personnes qui, pour des raisons professionnelles, sont amenées à manipuler régulièrement des sources radioactives ou à travailler au voisinage de telles sources (« travailleurs directement ou indirectement exposés »), 


\section{TABLEAU I}

Suspicions d'irradiation des travailleurs directement ou indirectement exposés selon trois classes professionnelles, en fonction du port d'un film dosimétrique (généralement de poitrine...) au moment de l'accident.

Distribution of directly and indirectly exposured professional workers in three categories, and presence or absence of physical dosimeter (generally chest dosimeter) at the time of suspected overexposure accident.

\begin{tabular}{|c|c|c|c|}
\hline 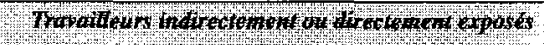 & avec dostinetre & sqpowothe & W1 \\
\hline Industrie non nucléaire & 9 & $9(50 \%)$ & 18 \\
\hline Professions de Santé, Enseignement et Recherche & 3 & $19(86 \%)$ & 22 \\
\hline Organisations nucléaires (EDF, COGEMA...) & 5 & $5(50 \%)$ & 10 \\
\hline Total & 17 & $33(66 \%)$ & 50 \\
\hline
\end{tabular}

(b) les personnes dont les activités (professionnelles ou privées) n'incluent pas naturellement l'usage de telles sources de rayonnements ionisants (« public »).

Trois classes peuvent en outre être distinguées dans la première catégorie (Tab. I) :

- les travailleurs de l'industrie, qui utilisent des moyens non destructifs pour vérifier la qualité de fabrication de certaines pièces, notamment la gammagraphie à l'aide d'iridium-192. Rentrent également dans cette catégorie les opérateurs intervenant dans la mise en place et l'utilisation d'accélérateurs linéaires ;

- les personnels des institutions de santé, d'enseignement et de recherche qui emploient fréquemment des sources radioactives, soit pour des travaux de recherches (diffractomètre, accélérateur linéaire), soit pour des applications médicales (radiothérapie) ;

- les agents de différents organismes nationaux liés au nucléaire (EDF, COGEMA, FRAMATOME, CEA...) pour lesquels il existe une grande variabilité des «accidents » en raison des nombreux métiers impliquant les rayonnements ionisants : les travaux sur matières radioactives et les opérations de nettoyage sont deux exemples parmi d'autres. Cette classe de travailleurs est néanmoins deux fois moins importante que les deux précédentes.

Les suspicions d'irradiation impliquant des personnes non exposées professionnellement ont été incluses dans la catégorie «public». Une ligne commune à l'ensemble des accidents est impossible à cerner en raison de la diversité de leur origine. Dans certains cas, ces personnes sont amenées à travailler ou à séjourner près d'une source de rayonnements ionisants en ignorant son existence. Dans d'autres cas, certaines d'entre elles croient avoir été exposées accidentellement, par exemple, en entrant dans une pièce d'irradiation contenant une source en fonction. D'autres cas concernent des personnes (journalistes, membres d'ambassade) ayant travaillé dans les territoires contaminés proches de 


\section{TABLEAU II}

Répartition des doses estimées par dosimétrie cytogénétique pour les différentes catégories de personnes impliquées.

The dose ranges estimated by biological dosimetry related to the repartition of the overexposured cases according to the different categories of implied people.

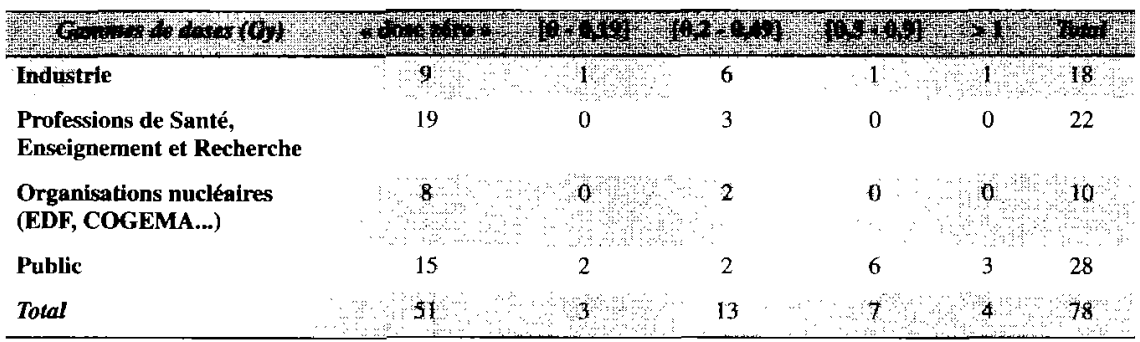

la centrale de Tchernobyl. Mais le plus grand nombre de cas expertisés par le LDBM dans cette catégorie implique une ou plusieurs personnes qui ont trouvé une source radioactive d'origine civile ou militaire, et l'ont gardée, soit sur elles, soit proche d'elles, sur une durée variable, de quelques secondes à plusieurs mois. Ces sources sont qualifiées d'《 orphelines ».

Pour des raisons de statistique, nous avons volontairement retiré deux grands accidents récents, celui survenu en 1998 en Géorgie et ayant impliqué 85 personnes et le second survenu à la fin de cette même année en Turquie et ayant impliqué dix personnes.

Les résultats des expertises peuvent, à leur tour, être divisés en deux grandes catégories (Tab. II) :

- les cas, heureusement majoritaires (51 cas des 78 examinés), où le nombre de dicentriques ( 0 ou 1) est compatible avec la fréquence observée dans une population normale ( 1 dicentrique pour 1500 cellules). Ces cas sont inclus dans le tableau II dans la colonne «zéro dose ». L'incertitude statistique de la méthode est proportionnelle au nombre de métaphases observées, et s'exprime par un intervalle de confiance à $95 \%$ de 0,2 Gy pour 500 cellules. Le nombre des cas apparaît relativement équilibré entre les différentes catégories ;

- les cas où le nombre de dicentriques est supérieur à cette fréquence minimale. Une grande proportion des doses estimées reste cependant inférieure à 1 Gy, avec une plus forte proportion du «public»par rapport aux travailleurs exposés. L'enquête sur les circonstances de l' « accident » révèle généralement que l'irradiation est aiguë et hétérogène, auquel cas la méthode utilisée sousestime les doses. Par exemple, lorsque seules les mains ont été exposées à des doses supérieures à 10 Gy (malheureusement fréquent lors d'un accident de gammagraphie), la dose estimée à partir de la fréquence des dicentriques dans 
les lymphocytes du sang périphérique ne dépasse pas 0,3 Gy. Par ailleurs, le type de courbe de référence utilisé peut avoir une importance notable sur l'estimation des doses, car la relation dose-effet dépend de la qualité du rayonnement et du débit de dose. Ceci se justifie particulièrement dans les accidents de criticité, où les effets biologiques relatifs des composantes du flux mixte neutron $/ \gamma$ sont nettement différents (Voisin et al., 1997). La comparaison avec la dosimétrie physique, dans les cas où celle-ci a été possible, montre d'ajlleurs un accord global avec l'estimation cytogénétique. Enfin, un laps de temps important (plusieurs mois) a dans certains cas séparé l'irradiation avérée de l'expertise. Dans ce cas, on sait que le taux de dicentriques diminue avec le temps d'autant plus vite que l'irradiation a été assez forte pour induire une lymphopénie (Bender et al., 1988 ; IAEA, 1986). Il est nécessaire de recourir à d'autres techniques cytogénétiques et notamment au dénombrement des aberrations chromosomiques stables (translocations, insertions) visualisées par hybridation in situ fluorescente (FISH). Cette technique, également développée au laboratoire, sort cependant du cadre de l'expertise des situations accidentelles récentes et ne sera pas décrite ici. Les résultats obtenus ont néanmoins été rapportés en détail par ailleurs (Sorokine-Durm et al., 1997).

On voit que, même si la dosimétrie biologique par cytogénétique conventionnelle n'est pas toujours capable d'estimer correctement la dose réellement reçue par la personne irradiée, elle permet de différencier les suspicions d'irradiation des irradiations avérées. Ceci est très important au plan humain, en raison de l'importante charge émotive liée aux circonstances de l'accident ainsi qu'à leurs conséquences éventuelles (assistance psychologique, perte d'emploi...).

\subsection{La dosimétrie cytogénétique en cas d'urgence radiologique}

Ainsi que cela a été rappelé dans l'introduction, une procédure particulière a êté adaptée de la technique standard, afin de répondre au problème du délai de réponse dans le cas d'un tri sur un grand nombre d'individus potentiellement irradiés. Les techniques de préparation et d'analyse des aberrations chromosomiques ont été simplifiées, et le nombre de métaphases observées réduit. L'observation de 50 métaphases permet d'assurer théoriquement un intervalle de confiance à $95 \%$ de \pm 1 Gy à l'estimation de dose. Cette marge d'incertitude semble satisfaisante aux équipes hospitalières en charge des irradiés, pour un premier tri.

Des tests sont effectués régulièrement en simulant une situation de crise au cours de laquelle plusieurs dizaines d'échantillons sanguins sont traitées simultanément. La détermination des doses est faite en aveugle sur des échantillons irradiés. Ces tests ont montré que l'incertitude statistique attendue était vérifiée pour toutes les doses (Fig. 2). 


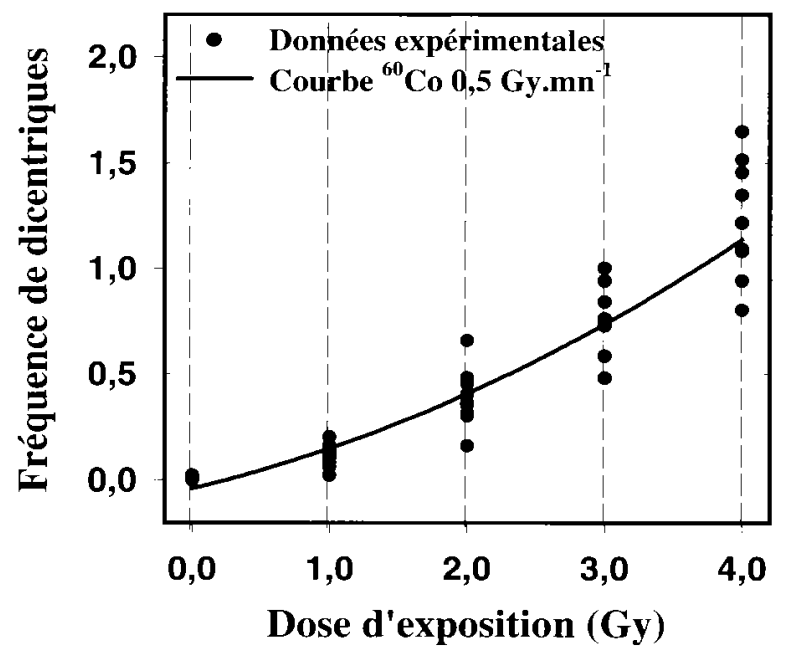

Figure 2 - Données expérimentales obtenues durant un exercice de simulation de crise, en employant la cytogénétique conventionnelle. 50 échantillons sanguins ont été traités suivant la procédure de

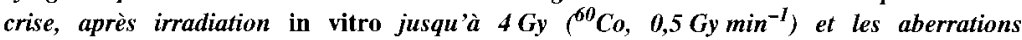
chromosomiques instables dénombrées sur la base de 50 métaphases de lymphocytes observées. La régression figure la courbe de référence du laboratoire établie dans les mêmes conditions expérimentales.

Experimental data of an emergency training during which 50 blood samples were in vitro irradiated up to $4 \mathrm{~Gy}\left({ }^{60} \mathrm{Co}, 0.5 \mathrm{~Gy} \mathrm{~min}^{-1}\right)$ and simultaneously analysed by conventional cytogenetics, based on the 50 metaphases reading. The biological dose estimates (in ordinate) is expressed according to the physical dose (in abscissa). The solid line represents the linearquadratic reference curve of the laboratory established in similar experimental conditions.

Néanmoins, la situation que l'on risque de rencontrer lors d'un accident réel, est intermédiaire entre la rapidité du tri et la précision de l'expertise. On a estimé la résoudre en traitant les échantillons sanguins de manière à assurer la lecture possible de 500 métaphases, mais en ne lisant que 50 dans une première étape. Une deuxième étape consiste à prolonger la lecture à 250 métaphases afin d'augmenter la précision générale d'un facteur $\times 2$, et ainsi de suite pour les cas les plus pertinents. Cette procédure a été appliquée après le deuxième accident radiologique de Géorgie, en 1998.

\subsection{Application à un cas réel : l'accident radiologique de Géorgie}

Fin juillet 98 , trois sources de ${ }^{137} \mathrm{Cs}$ (entre 0,17 et $150 \mathrm{GBq}$ ) ont été retrouvées enfouies dans le sol à proximité et dans le village de Matkhoji, situé à $300 \mathrm{~km}$ à l'ouest de Tbilissi. Elles provenaient d'une ancienne base militaire russe, 
abandonnée depuis 1992 et ayant brûlé depuis qui sert maintenant de terrain de jeux et de pâturage. À la demande du ministère de l'Environnement géorgien, puis de l'AIEA, une mission, constituée de 4 personnes de l'IPSN et d'un représentant de l'AIEA, s'est rendue sur place du 12 au 17 octobre 1998. Cette mission a permis de recueillir des informations sur les circonstances de l'exposition, de réaliser des prélèvements sanguins auprès de la population concernée pour une analyse sur place de certains paramètres hématologiques et de ramener des échantillons sanguins en vue d'une dosimétrie biologique.

À partir de l'enquête réalisée, trois groupes «critiques » ont été déterminés : enfants de plus de cinq ans, adultes de moins de 50 ans fréquentant le site, membres de la famille qui possédaient une source dans leur étable. L'analyse hématologique effectuée sur place (numération-formule sanguine) n'a montré aucune anomalie particulière.

Dès le retour de la mission en France, les échantillons sanguins provenant de 85 personnes ont été immédiatement traités. La phase de tri rapide, destinée à vérifier si une ou plusieurs de ces personnes présentait des signes évidents d'irradiation, a été achevée en sept jours sur la base de 50 cellules observées par échantillon. Des dicentriques ont été mis en évidence chez trois personnes seulement pour une dose globale n'excédant pas $0,5 \mathrm{~Gy}$ en moyenne. Une procédure d'expertise plus complète a suivi, sur la base de 250 cellules par prélèvement sanguin. Deux mois et demi ont été nécessaires pour observer plus de 22000 cellules chez les 85 personnes. Finalement, 30 dicentriques ont été dénombrés chez seulement 17 patients géorgiens. La dose la plus forte au corps entier estimée était de 0,3 Gy. Une majorité de ces dicentriques a été trouvée chez les enfants ayant joué à plusieurs reprises sur le terrain où se trouvaient les sources radioactives. Malheureusement, les informations fournies par la population étaient trop fragmentaires pour permettre une reconstitution plus efficace des doses reçues.

\subsection{Le test des micronoyaux pour les suspicions d'irradiation}

Il faut dénombrer les micronoyaux dans 1000 lymphocytes binucléées au minimum, pour obtenir un intervalle de confiance de la mesure du même ordre de grandeur que celui obtenu en dénombrant les dicentriques dans 500 métaphases. La vitesse d'observation dépend naturellement de la richesse de la préparation en cellules binucléées et en micronoyaux, mais le comptage peut être achevé en 1 à 2 heures environ. Cette rapidité d'observation, par rapport aux 2 jours nécessaires pour le dénombrement manuel des dicentriques dans les 500 métaphases, apparaît comme l'un des intérêts majeurs de cette méthode. De plus, ce test peut être mis en pratique par des opérateurs non spécialisés en cytogénétique. Cependant, 
on rencontre avec les micronoyaux la même limitation qu'en cytogénétique conventionnelle (intégration des doses au corps entier, disparition dans le temps, sous-estimation des irradiations hétérogènes, etc.). Quoique l'emploi des micronoyaux pour les expertises d'irradiation soit récent, les résultats sont globalement similaires à ceux donnés par les dicentriques. Une application intéressante est l'accident d'Istanbul, en 1998.

En 1998, deux sources de ${ }^{60}$ Co à usage médical (dont l'une d'activité 3,3 TBq) devant partir en réforme, sont entreposées à Istanbul dans un bâtiment inoccupé pendant près de 9 mois. Leurs containers sont alors vendus par erreur à des ferrailleurs d'Istanbul qui commencent à les démanteler en décembre 1998. Un syndrome aigu d'irradiation est diagnostiqué chez 10 adultes avec une forme particulièrement grave pour 5 d'entre eux. Dès la découverte de l'accident, l'AIEA contacte quatre laboratoires experts en dosimétrie cytogénétique : le Cekmece Nuclear Research and Training Centre (CNAEM, Turquie) ; l'IPSN (France); le National Radiological Protection Board (NRPB, Grande Bretagne) et le Leyden University Medical Center (LUMC, Pays-Bas).

Les échantillons sanguins provenant des 5 patients turcs les plus atteints sont traités à l'IPSN pour les aberrations chromosomiques instables (dicentriques, anneaux centriques et fragments) et stables (translocations, insertions) ainsi que les micronoyaux. Le mode de culture prolongé des lymphocytes pour obtenir des micronoyaux impose l'idée que l'on obtient ainsi une information partiellement corrigée pour les cas d'exposition chronique. Ainsi, les doses estimées en utilisant la courbe de référence du laboratoire sont tout à fait en accord avec celles obtenues avec les dicentriques, après correction pour prendre en compte une exposition chronique de 7 heures. La description complète de l'accident fait l'objet d'une publication AIEA en cours de rédaction.

\subsection{Adaptation du test des micronoyaux au tri d'urgence}

Comme pour la cytogénétique conventionnelle, la technique des micronoyaux a été adaptée pour le tri des irradiés en cas d'accident de grande envergure. Une méthode spécifique a été mise au point en diminuant substantiellement la quantité de sang nécessaire par culture et en améliorant le traitement des cellules afin de pouvoir observer un nombre suffisant de cellules binucléées - et donc de micronoyaux - même pour des doses d'irradiation fortes (4 Gy et au-dessus). 500 cellules binucléées peuvent être observées dans le temps nécessaire pour observer les dicentriques dans 50 métaphases. La plus grande vitesse de dénombrement des micronoyaux compense l'allongement du temps de culture nécessaire à leur obtention, ce qui fait que la durée totale de l'expertise est, dans les mêmes conditions expérimentales, d'une demi-journée seulement plus longue 


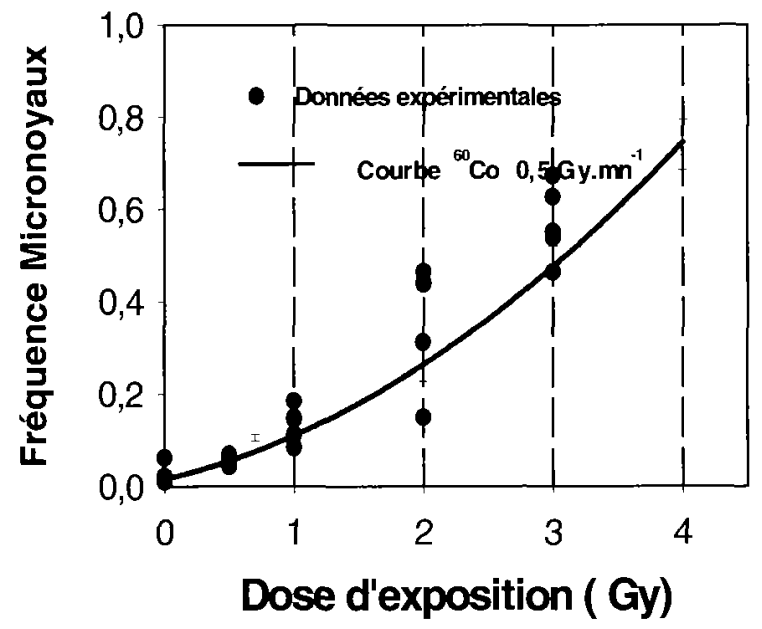

Figure 3 - Données expérimentales obtenues durant un exercice de simulation de crise, en employant le test des micronoyaux. 50 échantillons sanguins ont été traités suivant la procédure de crise, après irradiation in vitro jusqu'à $4 \mathrm{~Gy}\left({ }^{60} \mathrm{Co}, 0,5 \mathrm{~Gy} \mathrm{~min}^{-1}\right)$ et les micronoyaux dénombrés sur la base de 500 lymphocytes binucléés observés. La régression figure la courbe de référence du laboratoire établie dans les mêmes conditions expérimentales.

Experimental data obtained during an emergency training, by employing the micronuclei test. Fifty blood samples were treated according to the procedure described in Table 2 and in the text,

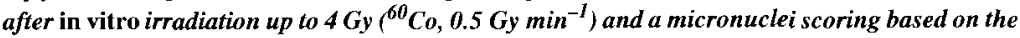
observation of 500 binucleated lymphocytes. The solid line represents the linear regression led through experimental points.

pour les micronoyaux que pour les dicentriques. Par ailleurs, la variabilité des résultats entre individus, plus importante pour les micronoyaux que pour les dicentriques, est compensée par la plus grande précision des mesures d'intervalle de confiance à $95 \%$ de $\pm 0,5$ Gy pour les micronoyaux par rapport à un intervalle de confiance à $95 \%$ de \pm 1 Gy pour les dicentriques (Fig. 3).

\section{Conclusion}

Nous avons confirmé dans cet article que, malgré ses limitations connues, le dénombrement des aberrations chromosomiques instables est utilisable dans de nombreux cas d'exposition accidentelle, supposées ou avérées, moyennant un certain nombre de précautions techniques. De plus, il est assez facilement adaptable au tri d'une population d'une centaine de personnes. Il faut cependant garder à l'esprit que, selon les circonstances d'exposition, les informations à retirer ne sont pas les mêmes. 
Nous avons également montré que les micronoyaux peuvent être utilisés à la place des dicentriques pour un tri de population, et qu'ils peuvent vraisemblablement être également utilisés pour certaines expertises accidentelles. Néanmoins, la formation de micronoyaux ne relève pas des mêmes mécanismes que la production de dicentriques, mais constitue une information complémentaire en ce qu'elle semble prendre en compte la radiosensibilité individuelle aux rayonnements ionisants. De plus, leur champ d'application semble plus restreint en ce qui concerne les faibles doses, l'exposition hétérogène ou fractionnée (Voisin, 1997). Une amélioration récente de la technique des micronoyaux consiste à augmenter sa spécificité en distinguant les micronoyaux d'origine mutagène de ceux d'origine clastogène par hybridation in situ fluorescente (Fimognari, 1997). Des expériences préliminaires effectuées au laboratoire ont permis de valider cette méthode. Nous nous attacherons dans un avenir proche à en vérifier l'aspect pratique.

\section{RÉFÉRENCES}

Bender M.A., Awa A.A., Brooks A.L., Evans H.J., Groer P.G., Littlefield L.G., Pereira C., Preston R.J., Wachholz B.W. (1988) Current status of cytogenetic procedures to detect and quantify previous exposure to radiation, Mutation Res. 196, 103-159.

Du Frain R.J., Littlefield L.G., Joiner E.E., Frome E.L. (1980) In vitro Human Cytogenetic DoseResponse Systems, The Medical Basis for Radiation Accident Prepardness, pp. 357-374.

Fimognari C. (1997) Analysis of radiation-induced micronucleoi by FISH using a combination of painting and centromeric DNA-probes, Mutagenesis 12,91-95.

International Atomic Energy Agency (1986) Biological Dosimetry: Chromosomal aberration analysis for dose assessment. Vienna, Technical report series $\mathrm{N}^{\circ} 260$.

Muller W.U., Streffer C. (1994) Micronucleus assay, Adv. Mutagen. Res. 129, I-134.

Paillole N., Voisin P. (1998) Is micronuclei variability a problem for overexposure dose assessment to ionising radiation? Mutation Res. 413, 47-56.

Perry P., Wolf S. (1974) New Giemsa method for the differential staining of sister chromatids, Nature 251, $156-158$.

Sorokine-Durm I., Durand V., Le Roy A., Paillole N., Roy L.,Voisin P. (1997) Is FISH-painting an appropriate biological marker for dose estimates of suspected accidental radiation overexposure? A review of cases investigated in France from 1995 to 1996, Environ. Health Perspect 105 (suppl 6), 1427-1432.

Voisin P., Durand V., Le Roy A., Paillole N., Chambrette V., Sorokine-Durm I. (1996) Chromosome aberrations of accidentally exposured persons in France: review of cases 1992-95, IRPA 9 Proceedings 3, 70-72.

Voisin P. (1997) Chromosome lesions as a short and medium term biological indicator of acute irradiation. Dans: L'homme blessé (L.A. Court, J. Lallemand, Eds.) pp. 139-150. Jouve, Paris.

Voisin P., Lloyd D., Edwards A. (1997) Chromosome aberrations scoring for biological dosimetry in a criticality accident, Rad. Prot. Dosim. 70, 467-470. 